\section{Monika Siegemund Rong}

\title{
Das Reizdarmsyndrom im Jugendalter
}

\author{
Therapieansatz mit PADMA LAX*
}

Das Reizdarmsyndrom (IBS) ist eine Erscheinung, die zwar mehrheitlich zwischen dem 30. und 50. Lebensjahr auftritt, jedoch häufig auch bei jüngeren Menschen diagnostiziert wird. Verschiedene Studien sagen, dass die Prävalenz bei Kindern und Jugendlichen zwischen 6 und 18 Jahren bei 6-14\% liegt, abhängig von der Art der Studie und der Region [1, 2]. Ätiologie und Pathogenese sind nach wie vor nicht vollständig geklärt und sehr komplex. Offenbar sind aber sowohl Umwelteinflüsse als auch genetische Faktoren an der Entstehung beteiligt, weshalb das IBS familiär gehäuft vorkommt [3]. Auch die Eltern-Kind-Interaktionen, sprich der Einfluss der Eltern bezüglich des Krankheitsverhaltens und des Umgangs mit Krankheitsbeschwerden, spielen eine Rolle. So leiden Kinder von Eltern mit IBS häufiger an gastrointestinalen und anderen Beschwerden, besuchen häufiger einen Arzt und fehlen öfter in der Schule [4]. Psychischer Stress und psychische Komorbiditäten exazerbieren ebenfalls die Symptomatik des IBS, und oft sind Angsterkrankungen und Depressionen bei Reizdarmpatienten vermehrt zu finden [5].

Wie bei Erwachsenen zielt die Therapie bei Kindern und Jugendlichen - nach korrekter Diagnosestellung - auf die Linderung der Hauptsymptome ab, wobei Gespräche und das Ernstnehmen der Ängste und Beschwerden der Patienten die Grundlage bilden. Wenn Stress oder seelische Belastungen als Mitauslöser für das IBS mit infrage kommen, so ist eine Kombination aus Psychotherapie und medikamentöser Therapie erwägenswert; ein Abwiegeln des Arztes mit den Worten «Das ist nur psychisch.»

*PADMED LAXAN ${ }^{\circledR}$ steht speziell dem Arzt zur Verfügung. Es ist kassenzulässig und identisch mit PADMA LAX ${ }^{\circledR}$. hingegen hilft den Patienten wenig [6]. Bei Vorliegen der obstipationsdominanten Form des IBS ist der Einsatz von Abführmitteln gerechtfertigt.

Folgender Erfahrungsbericht einer 23-jährigen Frau zeigt deutlich, wie sehr die Symptomatik des IBS die Lebensqualität insbesondere sehr junger Menschen über Jahre beeinträchtigen kann. Eine Odyssee von Behandlungsversuchen konnte schliesslich mit Akupunktur in Kombination mit dem Tibetischen Arzneimittel PADMA LAX ${ }^{\circledR}$, das sich aus 15 pflanzlichen und mineralischen Komponenten zusammensetzt, beendet werden. Der laxierende Effekt basiert auf anthrachinonhaltigen Pflanzen und $\mathrm{Na}$ triumsulfat, während Bitterstoffe und ätherische Öle sekretionsfördernd, karminativ und spasmolytisch wirken. $\mathrm{Zu}-$ sätzlich mildern Gerbstoffe Entzündungen und schützen die Schleimhaut. PADMA LAX kann bereits Kindern ab 6 Jahren verabreicht werden.

Bei der Patientin handelte es sich um eine junge, schlanke Frau mit Jahrgang 1987, die seit dem 12. Lebensjahr an der Diagnose Colon irritabile mit chronischer Obstipation litt. Zusätzlich bestand seit 3 Jahren der Befund einer chronischen Zystitis. Zum vorherrschenden Symptomenkreis gehörten vor allem stärkste Verstopfung, Blähungen, abdominelles Aufgedunsensein, Bauchschmerzen und Übelkeit. Über Jahre hinweg wurden diverse Therapien ausprobiert, von synthetischen Abführmitteln über Feigen, Trinken von warmem Wasser am Morgen, Ballaststoffe, mechanische Therapien wie Bauchmassage usw.

Da keine dieser Massnahmen den gewünschten Erfolg erzielte, wurde im Mai 2010 die Therapie mit PADMA LAX, kombiniert mit Akupunktur zur Stärkung des Nieren-Chi, begonnen. Bereits nach dreiwöchiger Behandlung besserte sich der Zustand, wobei sich der Schweregrad der Obstipation innerhalb von insgesamt 2 Monaten von 3 (starke Beschwerden) auf 0 (beschwerdefrei) verbesserte. $\mathrm{Zu}$ Beginn wurde das Arzneimittel mit 0/0/2 dosiert, wobei dank gutem Stuhlgang und normaler täglicher Stuhlfrequenz die Dosis nach 2 Wochen auf 0/0/1 reduziert werden konnte. Dieses Ergebnis führte auch zur Reduktion sämtlicher bisherigen Therapien und Massnahmen. Die Kombination aus Chinesischer und Tibetischer Medizin stoppte schliesslich auch die Blasenentzündungen. Die Patientin hat PADMA LAX sehr gut vertragen und äusserte eine sehr hohe $\mathrm{Zu}$ friedenheit mit dem Arzneimittel. Ihre Lebensqualität steigerte sich innert kürzester Zeit massiv.

Dank der vielfältigen Zusammensetzung von PADMA LAX steht somit ein Arzneimittel zur Verfügung, das nicht nur als reines Abführmittel die Verstopfung beheben kann, sondern durch vielfältige pflanzliche Sekundärstoffe eine regulierende Wirkung auf die gesamte Verdauung und die verschiedenen Darmfunktionen ausübt. Daher ist dieses Arzneimittel eine sinnvolle Therapieoption gerade für Menschen mit obstipationsdominantem Reizdarm oder chronischer Obstipation.

\section{Literatur}

1 Dong L, et al: Children in China: a schoolbased study. Pediatrics 2005;116:e393-e396.

2 Hyams JS, et al: Abdominal pain and irritable bowel syndrome in adolescents: a communitybased study. J Pediatr 1996;129:220-226.

13 Morris-Yates A, et al: Evidence of a genetic contribution to functional bowel disorder. Am J Gastroenterol 1998;93:1311-1317.

4 Levy RL, et al: Intergenerational transmission of gastrointestinal illness behavior. Am J Gastroenterol 2000;95:451-456.

5 Drossman DA, et al: AGA technical review on irritable bowel syndrome. Gastroenterology 2002;123:2108-2131.

6 ACG: ACG Releases Evidence-Based Systematic Review on Management of Irritable Bowel Syndrome. Bethesda, American College of Gastroenterology, 2008.
Dr. med. Monika Siegemund Rong

Islergasse 3, 8703 Erlenbach, Schweiz

Tel. +41 449916868 